\title{
End To End QoS Mapping Between Metroethernet and WiMAX
}

\author{
Leoncio Regal Dutra, Georges Amvame Nze, Cláudia J. Barenco Abbas, \\ Carlos Bon and Luciana Gomes.
}

\author{
${ }^{1}$ Universidade de Brasilia. \\ \{georges, leoncio\}@redes.unb.br. \\ ${ }^{2}$ Universidad Simón Bolívar. \\ barenco@1dc.usb.ve. \\ ${ }^{3}$ Serviço Federal de Processamento de Dados \\ \{carlos.bon, luciana.gomes\}@serpro.gov.br.
}

\begin{abstract}
This work aims the implementation and analysis of an environment formed by WiMAX and MetroEthernet networks. WiMAX offers unwired broadband access with high capacity of data transmission for dispersed areas. This network allows the interconnection of MetroEthernet networks with connection up to $10 \mathrm{Gbps}$. The study herein presented deals with a proposal of an end-to-end Quality of Service (QoS suitable not only for voice and video traffics but also for data traffic). Until the elaboration of this work, as far as we know, there is not a theoretical and practical study of the characteristics of real time traffic in WiMAX interconnecting MetroEthernet networks.
\end{abstract}

\section{Introduction}

Brazil is witnessing a radical change for network connections in metropolitan environment for public and research agencies. Twenty seven metropolitans networks will be created, one for each capital, where public and research organisms will divide modern optical infrastructure of high transmission capacity.

Although not being widely used in the market, the IEEE 802.16 network standard came to revolutionize the industry of wireless broadband access. It will offer ample transmission coverage for agricultural and metropolitans areas, with or without line of site. Such standard, known as WiMAX (Worldwide Interoperability Microwave Access), is defined by the IEEE group that deals with broadband access in dispersed areas. Although WiMAX does not create a new market, it should allow financial costs reduction and increase wireless communication usability. WiMAX sufficiently surpasses IEEE 802.11 limitations, such as bandwidth provision with the use of strong cryptography for data transmission.

Please use the following format when citing this chapter:

Dutra, I., R., Nze, G. A., Barenco Abbas, C. J., Bon, C., Gomes, I.,, 2008, in IFIP International Federation for Information Processing, Volume 265, Advances in Ad Iloc Networking, eds. Cuenca, P., Gucrrero C., Puigjaner, R., Serra, B., (Boston: Springer), pp. 1-12. 
With this scenario and the increasing demand for bandwidth to send and receive data, video, voice and television signal, an infrastructure with end-to-end Quality of Service (QoS) mapping is being proposed.

As the present scenery is to be heterogeneous at link layer, the implementation of an end-to-end QoS mapping is of great importance between both topologies. If one wants to guarantee a good end-to-end service, any kind of delay, delay variation (jitter) and data loss rate should obey strong QoS recommendation and implementation.

The topology herein presented includes link and network layer solutions, for effective end-to-end QoS guaranties, using IEEE 802.1p standard for frame priority in MetroEthernet and WiMAX QoS metrics for packet priority respectively. The overall end-to-end QoS would then be provided by the integration of WiMAX and MetroEthernet.

In this work we propose the implementation of QoS in MetroEthernet and WiMAX, based on measurements taken in laboratory and, suggested as a guide for future heterogeneous network implementations.

\section{Diffserv}

DiffServ is the abbreviation of Differentiated Services and is a technology that is scalable as to prevent the maintenance of flow state information inside the network.

At layer 3, inside and between networks, all flows having QoS guarantees are treated differently from other flows inside. A DS (Differentiated Services) field is present in the IP header protocol for a QoS metrics previously added by a border network node.

One of DiffServ's main functions is related to its behavior in analyzing a flow hop by hop (per-hop behaviors - PHB) inside a network. This behavior is the description of the treatment that is given to flows accordingly to their value mapped in the field DS (Differentiated Services).

PHB groups are implemented in each network node and are based essentially in scheduling and queuing management mechanisms.

The DiffServ model redefined the TOS (Type of Service) octet in the IPv4 header as DS octet. It contains two fields: the DSCP (Differentiated Services Code Point), with 6 bits, used in the determination of the PHB and two CU (Currently Unused) bits reserved for future use. Both fields must be ignored for the purpose of PHB election. Figure 1 shows what has been described previously.

\begin{tabular}{|lllll|lll|}
0 & 1 & 2 & 3 & 4 & 5 & 6 & 7 \\
\hline \multicolumn{4}{|c}{ DSCP } & & & \multicolumn{2}{c|}{$\mathrm{CU}$} \\
\hline
\end{tabular}

Fig. 1. DS byte structure in IP header.

DiffServ defines two PHB groups: Assured Forwarding [4, 12, 14] and Expedited Forwarding [4, 12, 14]. 
End To End QoS Mapping

Between Metroethernet and WiMAX 3

- $\mathrm{PHB}-\mathrm{AF}$

- The AF group aims to supply distinct traffic class with varying levels of loss probability. For such, it praises the existence of four distinct AF classes, each one with proper resources attributed and treated independently. Inside each class there are three levels of loss precedence that correspond to a greater or minor loss probability inside the class queue.

- $\mathrm{PHB}-\mathrm{EF}$

- The EF group is defined as a unique class with express forwarding. The EF PHB must be implemented when there is a necessity to transmit a traffic profile with low loss, low delay, little variation in the delay (low jitter) and guaranteed bandwidth. The EF PHB is used to identify and direct multimedia application traffic such as real time voice and video.

\section{$3 \quad 802.1 p$}

To guarantee the transmission of information with good characteristics related to delay and its variation, as well as data loss rate, the $802.1 \mathrm{p}$ protocol can assist all type of traffic with QoS requirements.

The $802.1 \mathrm{p}$ specification introduces new mechanisms for traffic priority in IEEE 802 networks to support critical traffic and dynamic multicast filtering. Traffic volume limitation is implemented in LAN (Local Area Network) switches using these mechanisms. Although they both are interesting in performance, the $802.1 \mathrm{p}$ priorities mechanism has a direct impact in IEEE 802 quality of service networks [16].

In IEEE 802.3 (Ethernet standard), there is no existence of a priority header field for traffic priority mapping. So for Ethernet networks, IEEE 802.1q should be used to support the VLAN identification. The 802.1q should include a field of three bits for assigned user priority, as for future traffic aggregation requiring QoS mechanisms.

The $802.1 \mathrm{p}$ priorities mechanism is implemented at link layer. To make use of this mechanism, switches must have the capacity of mapping the traffic to different classes. This protocol defines eight priorities values, leaving to the network administrator the task to attribute values to the different types of traffic flows in the network.

Priority 7 mapping is the highest value raised and must carry critical traffic to the network, such as route update. Priorities 5 and 6 are indicated for applications sensible to the delay, such as voice and video. In turn, class 4 and 1 can carry from controlled load to low priority traffic.

Class 0 specifications are similar to best effort for packets with no special priority. Equipments do not have to implement eight different types of queuing disciplines on each port for the system to work properly $[14,15]$.

In Table 1, the recommended mapping is demonstrated for priority values that should apply for user applications and the queue it should hold to. This recommendation must be in agreement with the number of queues available in the equipment. 
Although there is a mechanism of traffic differentiation capable of rearranging packets and guaranteeing a high priority delivery for critical applications, the $802.1 \mathrm{p}$ by itself cannot give any guarantee of how much latency has been introduce. The $802.1 p$ becomes inadequate for applications that need rigid guarantees of such parameter. However, if used jointly with other QoS mechanisms, for example those implemented at network layer, the $802.1 \mathrm{p}$ can be vital in the integration of traffic differentiation in the same network [17].

Table 1. Mapping between the priority

value and wait queue for different numbers of wait queue.

\begin{tabular}{c|c|c|c|c|c|c|c|c|c}
\hline & \multicolumn{8}{c}{ Available number of Classes of Traffic } \\
\cline { 2 - 12 } & & $\mathbf{1}$ & $\mathbf{2}$ & $\mathbf{3}$ & $\mathbf{4}$ & $\mathbf{5}$ & $\mathbf{6}$ & $\mathbf{7}$ & $\mathbf{8}$ \\
\hline & $\mathbf{0}$ & 0 & 0 & $\mathbf{0}$ & 1 & 1 & $\mathbf{1}$ & 1 & 2 \\
\cline { 2 - 12 } & $\mathbf{1}$ & 0 & 0 & 0 & 0 & 0 & 0 & 0 & 0 \\
\cline { 2 - 11 } & $\mathbf{2}$ & 0 & 0 & 0 & 0 & 0 & 0 & 0 & 1 \\
\cline { 2 - 11 } & $\mathbf{3}$ & 0 & 0 & 0 & 1 & 1 & 2 & 2 & 3 \\
\cline { 2 - 11 } & $\mathbf{4}$ & 0 & 1 & 1 & 2 & 2 & 3 & 3 & 4 \\
\cline { 2 - 11 } & $\mathbf{5}$ & 0 & 1 & 1 & 2 & 3 & 4 & 4 & 5 \\
\cline { 2 - 11 } & $\mathbf{6}$ & 0 & 1 & 2 & 3 & 4 & 5 & 5 & 6 \\
\cline { 2 - 10 } & $\mathbf{7}$ & 0 & 1 & 2 & 3 & 4 & 5 & 6 & 7 \\
\hline
\end{tabular}

\section{WiMAX Quality Of Service}

To define the priority of MAC SDUs (Medium Access Control Service Data Unit) through the existing connections, each connection (defined as CID - Connection ID) is mapped in a daily pre-define class of scheduling. Each class has a set of parameters that quantifies QoS prerequisites. These parameters are managed through the management messages type DAS (Dynamic Service Addition) and DSC (Dynamic Service Change). Four classes of services are presented for QoS metrics implantation in WiMAX $[3,18$ and 19]:

- Unsolicited Grant Service (UGS) - designated for real time traffic with constant rate flow, such as emulations circuits and ATM CBR.

- Real Time Polling Service (rtPS) - designated for real time traffic with variable rate flow rate (variable packet size), such as MPEG video.

- Non-Real-time Polling Service (nrtPS) - designated for stored traffic requiring low delay with variable flow rate (variable packet size), such as stored MPEG video.

- Best Effort (BE) - designated for variable traffic rate (variable packet size), such as TCP/IP. 
End To End QoS Mapping

Between Metroethernet and WiMAX 5

\section{QoS Mapping}

From the techniques herein presented, the most significant scheduling mechanism defined for the IEEE 802.16 standard, was defined by Hawa [1 and 2]. His work presented a random analysis of scheduling mechanism based on the Fair Queuing technique with QoS support.

Another possibility in applying Quality of Service to IEEE 802.16 is using DiffServ. In networks implementing DiffServ, the admission control is based on Bandwidth Brokers (BBS) and Service Level Agreement (SLA) mechanisms. This solution does not fix the control flow congestion problem, where all flows having the same classification can be degraded. Admission Control solutions could be used to fix this problem.

The solution proposed by LCT - UC (Laboratory of Communications and Telemetric of the University of Coimbra) uses a metric to calculate an index of congestion of the network element to verify if it can admit a new flow $[4,5,6,7$ and 8].

There are some techniques of probing the network using Packet Probing [9, 10 and 11] but they do not have applicability to a generic architecture of Quality of Service. Such conclusion elapses from the fact that they only can be applied on an IEEE 802.16 network, or either, they only deal with the possibilities to provide QoS in an IEEE 802.16 network.

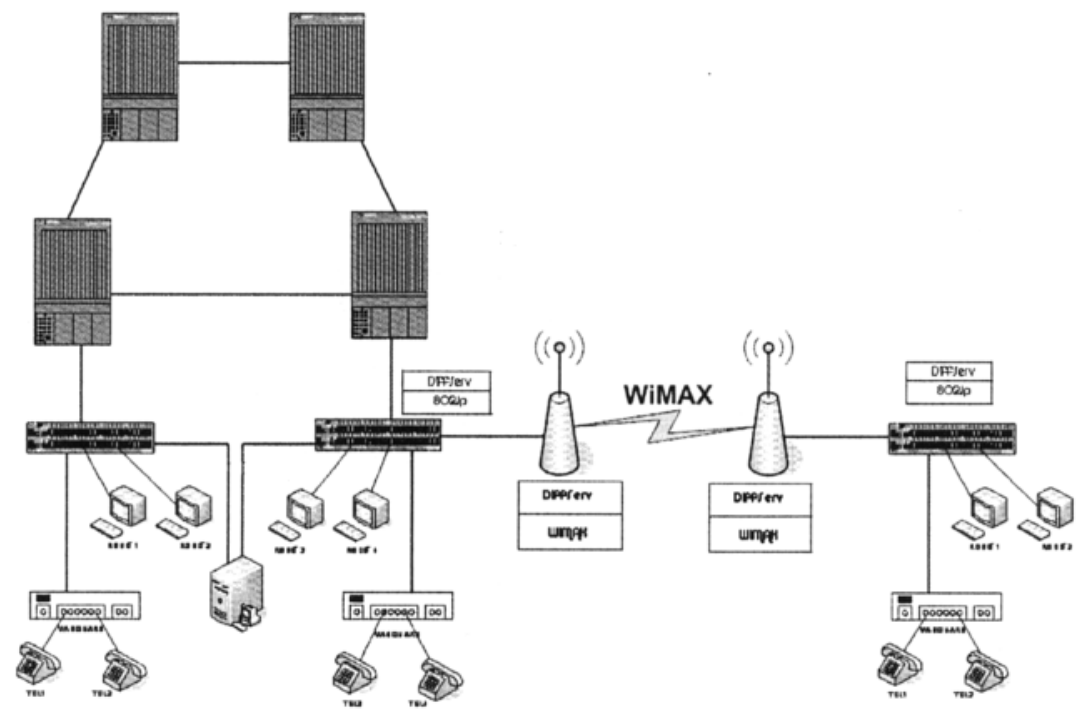

Fig. 2. Architecture of MetroEthernet - WiMAX networks.

These problems led us to consider a generic architecture of QoS, based on DiffServ, IEEE $802.1 \mathrm{p}$ and 802.16 standards, where MetroEthernet networks are 
connected through WiMAX wirelessly as shown in Figure 2, to extend the Metropolitan Area Network (MAN).

The proposal defined in this work, on one side, uses IEEE 802.1p as a mapping model defining link layer priorities and on the other, a group of DiffServ PHBs and Class of Services defined in IEEE 802.16. The proposal includes a static mechanism of admission control capable of reflecting the network state, guaranteeing all requirements considered essential for networks that implement QOS mechanisms. Requirements such as end-to-end delay, jitter and packet loss reduction.

The IEEE 802.1p standard implements 7 types of priorities, from the lowest to the highest priority (1...7), where each priority has an individual applicability.

To implement a QoS mapping between IEEE 8021.p and DiffServ (DSCP - COS) a ACLs (Extended Access List) will be used to maintain the priority parameters. These ACLs contain a table, where the DSCPs codes and their respective classifications are listed. The related list contains from 0 to 63 possibilities of PHBs mapping. Through this priority, is made the DSCP to COS mapping, as shown at Table 2 [12].

As an example, a packet having its DSCP value mapped to 6 will imply in having a packet mapped to class 6 by the IEEE $802.1 \mathrm{p}$ protocol.

In implementation terms, the support for MP3 mapping relies on the capacity to transmit other traffic aggregates, through priorities mechanisms. However, a limit for traffic debit must exist to prevent traffic congestion caused by other traffic aggregate. The packages that exceed this limit will have to be eliminated.

Table 2. Rule of Mapping 802.1p - DiffServ - WiMAX.

\begin{tabular}{c|c|c|c|c}
\hline $802.1 \mathrm{p}$ & PHBs & Diffserv & WiMAX & New Mapping \\
\hline 0 & $0-7$ & $\mathrm{BE}$ & $\mathrm{BE}$ & MP1 \\
\hline 1 & $8-15$ & - & - & \\
\hline 2 & $16-23$ & $\mathrm{BE}$ & $\mathrm{BE}$ & \\
\hline 3 & $24-31$ & $\mathrm{AF}$ & $\mathrm{nrtPS}$ & MP2 \\
\hline 4 & $32-39$ & - & - & \\
\hline 5 & $40-47$ & $\mathrm{EF}$ & rtPS & \\
\hline 6 & $48-55$ & $\mathrm{EF}$ & UGS & MP3 \\
\hline 7 & $56-63$ & - & - & \\
\hline
\end{tabular}

MP2 mapping is a little different from MP3, supplying only one type of service at the moment of congestion in the network. In other words, the use of MP2 will have minimum delay, jitter and packet loss guarantees at the moment of network congestion. Even tough, these guaranties cannot be enough to comply with the recommended ITU-T QoS requirements [13].

In case of a network congestion, MP2 mapping will be applied to those packets relying on higher priorities recommendation and so, providing a higher probability to be delivered.

Table 2 shows two levels. For priority issues, where the COS is equal to 6 for example, traffic of higher importance will be mapped as more sensible to delay, jitter and packet loss. 
For other types of network traffic sharing the same bandwidth, such as best effort, a MP1 mapping will be used.

\section{The TestBed}

The environment of development and chosen test is based on personal computers (PC) with the operational system Windows, configured with applications to generate traffic (called PC1, PC2, PC3 and PC4). Moreover, the architecture is composed for equipment such as switches and routers that will be shown in Figure 3.

An item of great importance in any system of quality test, is the traffic generator software, that must be capable to generate traffics with Internet flows characteristics and with support for QoS. These components are computers PC1, PC2 and PC4.

The receiving application will have to be capable of receiving the packets with no delay, so that the receiving interval of time does not affect the final results.

It will also have, to allow the attainment of statisticians who make possible the performance evaluation of the network, with respect to delay, jitter, packet loss percentage and data rate reception (throughput), $\mathrm{PC} 3$ being responsible for executing this function.

The network 1 is composed of two personal computers (PC1 and PC2), plugged in proper switch and router. The computers are connected to an interface with $1 \mathrm{Gbps}$ and full-duplex connection. The router has $5 \mathrm{Mbps}$ - Full-Duplex of speed connection.

In the Network 2, switches are connected by two Pre-WiMax antennas, having a speed relatively low data transmission rate (something close to 3,5 Mbps HalfDuplex). It is important to say that all these values are difficult to the found in a MetroEthernet and WiMAX network standards, since the tests are carried through a test environment and not in a real world environment.

In relation to the antennas Pre-WiMAX, it is important to detach the item of configuration of them. Initially, an equal percentage of use of band for uplink and downlink was defined, that is, $50 \%$ of use for uplink and $50 \%$ of use for downlink. Later, the maximum tax supported of traffic for uplink and downlink was defined that it is of $20000 \mathrm{Kbps}$ for each one. In case that the traffic is bigger of what the supported one, a size of buffer was also configured to store these packages. The size of the buffer is of $20000 \mathrm{kbits}$ equally for uplink and downlink.

In the antenna a scheduling algorithm was configured, its proper implemented in the hardware, to prioritize channels that will treat better, all the packages marked with priority. Of this form, all the packages marked with COS up to 3 are considered by the antenna as a traffic of low priority, and all the packages marked with COS of 4 the 7 , are considered by the antenna as a traffic of high priority.

It is important, the implementation of DiffServ Model in the antennas, to differentiate the packages marked with quality of service or not. In this implementation, packages without priorities will go to be treated by the PHBs with values between 0 and 7 (MP1). For mapping MP2, the packages they will be treated by the PHBs with values between 24 and 31 and for the mapping MP3, values between 48-55. With this we apply the Model of Diffserv Service in the antennas PreWIMAX. 


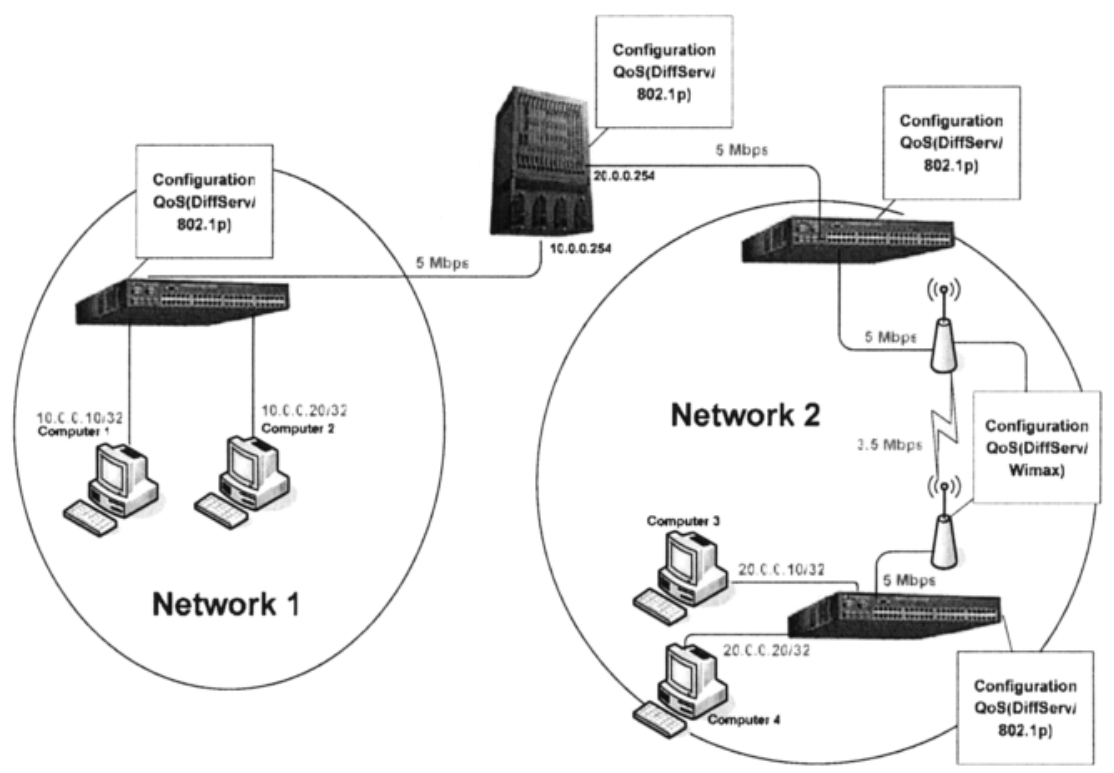

Fig. 3. Architecture of the Atmosphere of Test.

The configuration of IEEE $802.1 \mathrm{p}$ in the equipment is carried through in all the components that the architecture of the network composes in accordance with the service contracted for the user. Switch of network 1 will be the responsible one for marking the packages that will be generated in the network with corresponding values its priority, being the packages marked with $3 \mathrm{COS}=$ referring to the model of Mapping MP2 and COS $=6$ for the Model of Mapping MP3.

The others switchs will have the functionality of mapping the packages of DSCP for COS applying with this the priorities defined in the Model of Mapping. For this the ACLs was configured in the equipment the priority using (Extend Access List). Through the ACL, the packages they will be mapped of DSCP - COS and transmitted for its lines of priorities.

The Infovia Brasilia specifies up to 30 simultaneous VoIP calls. Of this form we configure the environment to support 30 simultaneous calls, that is, 30 traffics of VoIP at the same time.

Figure 4 represents the functioning of the mapping considered in the work.

In environment MP1, two standards of traffic had been defined: 1 VolP and 1 competitor, in accordance with table 3. 
End To End QoS Mapping

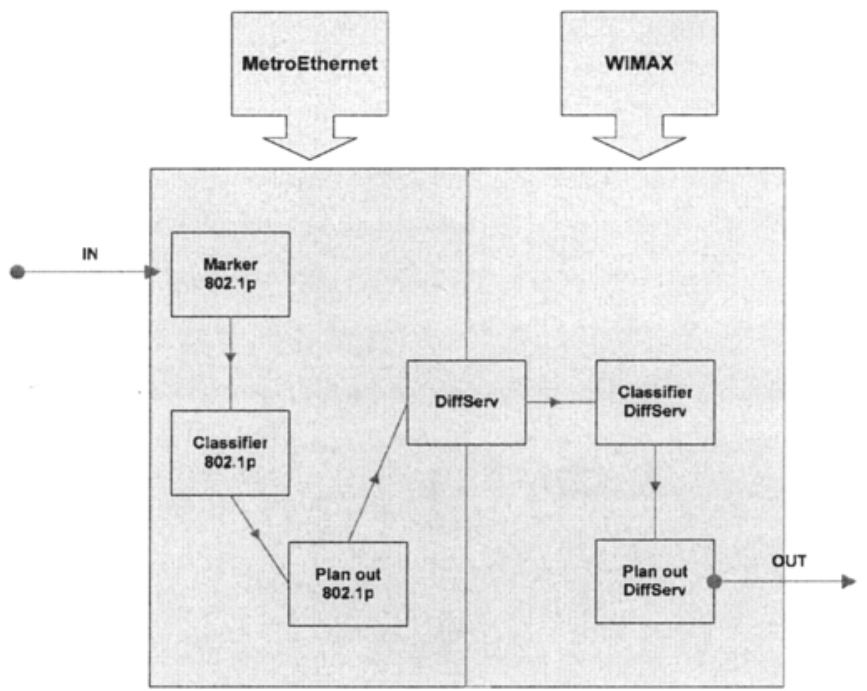

Fig. 4. Mapping 802.1p_DiffServ_Wimax.

Table 4. it shows the traffic defined for Mapping MP2. We continue with the two standards of traffic, only prioritizing the VoIP traffic.

Table 5. it shows the traffic defined for Mapping MP3. In this in case, traffic of VoIP is mapped with a higher priority.

Table 3. Flow of Traffic for MP1.

\begin{tabular}{c|c|c}
\hline Speed & Class & Siza \\
\hline $64 \mathrm{Kbps}$ & MP1 & 232 bytes \\
\hline $1000 \mathrm{Kbps}$ & MP1 & 1024 bytes \\
\hline
\end{tabular}

Table 4. Flow of Traffic for MP2.

\begin{tabular}{c|c|c}
\hline Speed & Class & Siza \\
\hline $64 \mathrm{Kbps}$ & MP2 & 232 bytes \\
\hline $1000 \mathrm{Kbps}$ & MP1 & 1024 bytes \\
\hline
\end{tabular}

Table 5. Flow of Traffic for MP3.

\begin{tabular}{c|c|c}
\hline Speed & Class & Siza \\
\hline $64 \mathrm{Kbps}$ & MP3 & 232 bytes \\
\hline $1000 \mathrm{Kbps}$ & $\mathrm{MP1}$ & 1024 bytes \\
\hline
\end{tabular}

For better visualization of the Mapping, since Mapping MP1 until the Mapping MP3, figure 5, figure 6 and figure 7, compare to delay, jitter and packet loss for all types of mappings. In this visualization, we observe a significant improvement of the 
10 Leoncio Regal Dutra, Georges Amvame Nze, Cláudia J. Barenco Abbas,

\section{Carlos Bon and Luciana Gomes.}

parameters of Quality of Service, delay, jitter and packet loss, for a VoIP traffic. In this way, we observe that the Model of Mapping MP1 and MP2 are not recommended, while the Model of Mapping MP3 is recommended for a VoIP traffic based on the recommendations of the ITU-T [13].

Delay (MP1, MP2, MP3)

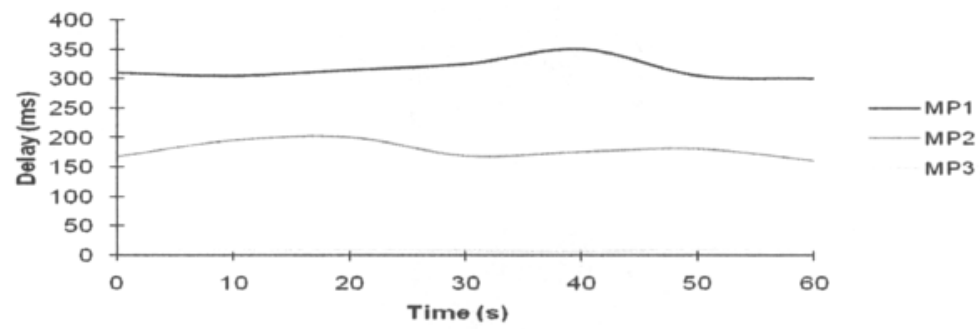

Fig. 5. Comparative of the Delay of Mapping (MP1, MP2, and MP3).

Jitter (MP1, MP2, MP3)

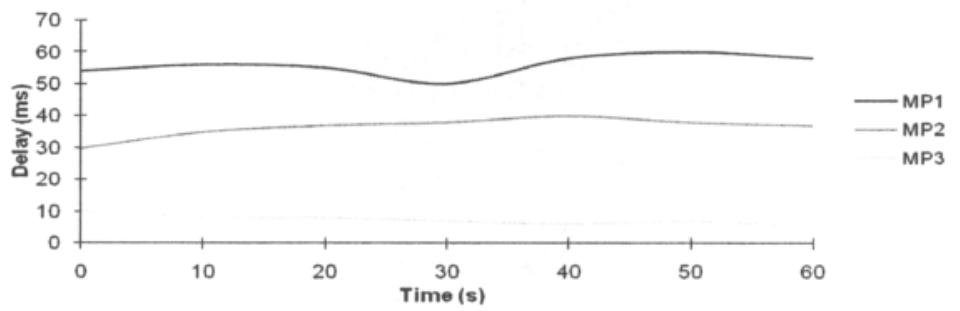

Fig. 6. Comparative of Jitter of Mapping (MP1, MP2 and MP3).

Packet Loss (MP1, MP2, MP3)

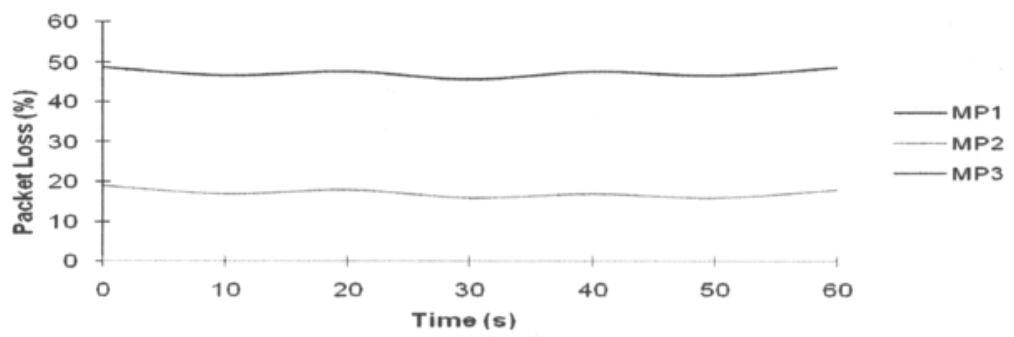

Fig. 7. Comparative of Packet Loss of Mapping (MP1, MP2 and MP3). 


\section{Conclusions and Future Works}

In relation to the initial objective to implement a Quality of Service in a MetroEthernet and WiMAX networks, it can be said that the results were widely reached, based in the recommendations of the ITU-T. A recommendation of the Model of Mapping MP3 for the environment of the testbed can be defined to VoIP applications. With the implementation of the MP3 mapping ones can take care of higher traffic application sensible to delay, jitter and packet loss. Our implementation brought innumerable advantages for the integration of the IEEE 8021.p with the parameters of quality of service, if a transmission of VolP of high quality has to be transmitted in the network, as proposed in this work.

Another advantage observed in this work is the integration of Diffserv as being the integrator in QoS mapping, since it is applied at the Network layer. DiffServ is much more flexible to the fact that it has great influence in the differentiation of any type of traffic service

The environment of tests, where exactly a small replica of the real world environment, called INFOVIA to build in Brasilia (Brazil) and, can be validated with all kind of QoS metric herein proposed.

In the near future, we can collect a real data information of the production environments and compare with the ones retrieved from the testbed environment.

Future works can give continuity to the improvement of the IEEE 802.16 QoS mapping and not by using a Pre-WiMAX equipment.

\section{Reference}

1. HAWA, M. Stochastic Evaluation of Fair Scheduling with Applications to Qualityof-Service in Broadband Wireless Access Networks. PhD's Thesis. Thesis University of Kansas. August 2003.

2. HAWA, M.; DAVID, W. Quality of Service Scheduling in Cable and Broadband Wireless Access Systems. In: Tenth International Workshop on Quality ofvService (IWQoS 2002). Miami, Florida, May 2002, pp. 247-255.

3. IEEE Std 802.16.2-2003. IEEE Standard for Local and metropolitan area networks. Part 16: Air Interface for Fixed Broadband Wireless Access Systems - Amendment 2: Medium Access Control Modifications and Additional Physical Layer Specifications for 2-11 GHz (Amendment to IEEE Std 802.16-2001). April 01, 2003.

4. G. Quadros, et al. "A QoS Metric for Packet Networks", in Proceedings of SPIE International Symposium on Voice, Video, and Data Communications Conference, Boston, USA, 1-5 Novembro, 1998.

5. G. Quadros, et al. "Measuring Quality of Service in Packet Networks", in Proceedings of the 2nd Conference on Telecommunications, Instituto de Telecomunicações (Portugal), Sesimbra, Portugal, 15-16 Abril, 1999.

6. QUADROS, et al. "Approach to the Dynamic Forwarding of Packets in a Differentiated Service Based Router", in Proceedings of SPIES Symposium on Voice, Video, and Data Communications conference on Quality of Service Issues Related to Internet II, Boston, USA, 19-22 Setembro, 1999. 
12 Leoncio Regal Dutra, Georges Amvame Nze, Cláudia J. Barenco Abbas, Carlos Bon and Luciana Gomes.

7. QUADROS, et al. "An Approach to Support Traffic Classes in IP Networks", in Proceedings of QofIS'2000 - The First International Workshop on Quality of future Internet Services, Berlin, Germany, 25-26 Setembro, 2000.

8. QUADROS, et al. "The Role of Packet-dropping Mechanisms in QoS Differentiation", in Proceedings of ICON'2000 - IEEE International Conferences on Networks, National University of Singapore, Singapura, 05-08Setembro, 2000.

9. BRESLAU, et al. "Endpoint Admission Control: Architectural Issues and Performance", in Proceedings of ACM SIGCOM 2000, Stockolm, Suécia, Agosto, 2000.

10. ELECK,et al. "Admission Control Based on End-to-End Measurements", in Proceedings of IEEE INFOCOM 2000, Tel Aviv, Israel, Março, 2000.

11. BIANCHI, et al. A migration Path to provide End-to-End QoS over Stateless networks by Means of a probing-driven Admission Control, Internet Draft, draftbianchi-blefari-end-toend-qos-01.txt, Julho, 2001.

12. HEINANEN, J. et al. "Assured Forwarding PHB Group", IETF RFC 2597, June 1999 .

13. Internation Telecommunication Union - ITU, Recomendações G.711, G.723.1, G.726, G.728, G.729, Acesso em 14/06/2007. http://www.itu.int.

14. RFC 2998 - A Framework for Integrated Services Operation over Diffserv Networks. Acesso em 01/04/2007. http://www faqs.org/rfes/rfc2998.html.

15. IEEE 802.1 P,Q - QoS on the MAC level. Acesso em 02/05/2007. http://www.tml.tkk.fi/Opinnot/Tik1 10.551/1999/papers/08IEEE802.10osIn MAC/qos.html.

16. A Bridging between IEEE 802.1Q VLANS. Acesso em 10/08/2007. http:/www.cisco.com/univercd/ce/td/doc/product/software/ios $12 \mathrm{~V} / 12$ lnewft/ $121 \mathrm{t} / 121 \mathrm{t} 3 / \mathrm{dtbridge}$.htm\#xtocid 114535

17. UBIK, S., VOJTECH, J. QoS in Layer 2 Networks with Cisco Catalyst 3550. CESNET Technical Report 3/2003. April 20, 2003.

18. DELICADO, j., BARBOSA, L.O., DELICADO, F, CUENCA, P. A QoS-aware protocol architecture for WiMAX. IEEE CCECCE/CCGEl, Ottawa, May 2006.

19. ALAVI, H.S., MOJDEH, M., YSZDANI, N. A Quality of Service Architecture for IEEE 802.16 Standards. Conference on Communications, Perth, Western Austraia, 35 October 2005. 